\title{
USANDO TWITTER PARA PROMOVER LA EDUCACIÓN CONTINUA Y LA INVESTIGACIÓN EN SALUD EN EL PERÚ
}

\section{[USING TWITTER TO PROMOTE CONTINUOUS EDUCATION AND HEALTH RESEARCH IN PERU]}

\author{
Walter H. Curioso ${ }^{1,2, a, b}$, Eduardo Alvarado-Vásquez ${ }^{1, c}$, \\ Renzo Calderón-Anyosa ${ }^{3, d}$
}

Sr Editor. La popularidad de las redes sociales se ha incrementado en los últimos años; sitios web como Facebook, MySpace, y Twitter permiten a los usuarios comunicarse, compartir información y mantener un sitio web personal con actualizaciones de datos, fotos, listas de amigos en línea y grupos de interés virtuales.

Twitter (www.twitter.com) es un servicio en línea lanzado en el 2006, que permite a los usuarios enviar y recibir mensajes cortos, máximo 140 caracteres, conocidos como tweets. Estos mensajes se muestran en la página de inicio del usuario y se entregan a todos los seguidores o followers del autor. Dichas actualizaciones pueden ser visualizadas en la web o mediante aplicaciones para computadores y dispositivos móviles.

El mundo científico no es ajeno a esta revolución de la información, y se evidencia en el creciente número de profesionales, investigadores, revistas científicas, universidades, centros de investigación, asociaciones científicas, entre otros, que han encontrado en Twitter una nueva manera para comunicarse y compartir sus puntos de vista sobre documentos publicados recientemente, presentaciones científicas, debates, así como información sobre becas, carreras y otros artículos de interés.

En Perú, el uso de Twitter no está muy difundido. Según la encuesta nacional de la Pontificia Universidad Católica del Perú, solo el $10 \%$ de los usuarios de Internet tienen una cuenta en Twitter (1). Por otro lado, no existen publicaciones sobre su uso en el campo de salud e investigación en el Perú. El objetivo de la

\footnotetext{
Facultad de Salud Pública y Administración "Carlos Vidal Layseca", Universidad Peruana Cayetano Heredia, Lima, Perú.

2 Biomedical and Health Informatics, School of Medicine, University of Washington, Seattle, WA, USA.

3 Facultad de Medicina "Alberto Hurtado", Universidad Peruana Cayetano Heredia, Lima, Perú.

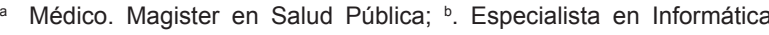
Biomédica; c. Médico Cirujano; d. Estudiante de medicina.
}

Recibido: 01-02-11 Aprobado: 02-02-11 presente carta es presentar algunas de las experiencias del uso de Twitter en la Universidad Peruana Cayetano Heredia (UPCH) para la promoción de la educación continua y la investigación en salud, además de discutir usos adicionales de este sitio web en el contexto de la salud pública.

Para la promoción de la educación continua, Twitter puede ser usada para difundir información sobre noticias de salud, conferencias, cursos, becas y otros recursos de interés, manteniendo a los usuarios actualizados y promoviendo la participación en estos eventos. Se pueden utilizar hashtags, o etiquetas de metadatos, que permiten agrupar los tweets por categorías o nombres específicos, lo cual es útil para encontrar de una manera más sencilla todos los tweets de un tema relacionado y participar en discusiones con personas de todo el mundo. El uso del hashtags permite, además, transmitir o seguir en tiempo real eventos como conferencias y cirugías, con la opción adicional de resolución de encuestas en vivo y preguntas directas a los ponentes.

Además, Twitter ha demostrado ser útil en programas de educación superior como herramienta de colaboración y discusión entre alumnos y docentes ${ }^{(2)}$, difusión de noticias e inclusive ha sido utilizado para la evaluación y retroalimentación sobre el desempeño de los docentes y cursos ${ }^{(3)}$.

Por ejemplo, el programa QUIPU (4) de la UPCH utiliza Twitter para promover las actividades de los miembros y de la Red QUIPU (@redquipu). En el Diplomado de Informática Biomédica de la UPCH se viene utilizando el hashtag: \#ibquipu para actualizar la información relacionada con los cursos, clases y conferencias del diplomado así como para intercambiar información y temas de discusión generados en el curso y recibir opiniones o comentarios de los seguidores.

Otras cuentas de la UPCH para la promoción de actividades de educación continua y diseminación de eventos son: @CayetanoHeredia (cuenta general UPCH), @faspaupch (Facultad de Salud Pública y Administración), @DURIN_UPCH (Dirección de Relaciones Internacionales), y @EDUCACION_UPCH (Facultad de Educación).

En el campo de la promoción de la investigación, cada vez es más común utilizar Twitter para mantenerse alerta sobre convocatorias de oportunidades de financiamiento para trabajos de investigación. Organismos internacionales como los Institutos Nacionales de Salud de los Estados Unidos (NIH), @NIHforFunding, actualiza convocatorias para becas de estudio, subvenciones, y disemina comentarios sobre publicaciones recientes. 
Revistas científicas como The Lancet (@TheLancet), PLoS ONE (@PLoSONE) o The New England Journal of Medicine (@NEJM) publican enlaces directos a sus artículos más recientes, dando a conocer las últimas investigaciones en los diversos campos de la salud.

A nivel local, la Oficina de Promoción a la Investigación de la UPCH ha creado la cuenta @opiupch que permite la diseminación de oportunidades de financiamiento, información relacionada con promoción a la investigación y trabajos realizados por investigadores, docentes y alumnos de nuestra institución. Este es un buen ejemplo del uso de las nuevas tecnologías como para promover la investigación científica en estudiantes, investigadores y profesionales de la salud.

Existen otras aplicaciones de Twitter en salud. Así, instituciones como la Organización Mundial de la Salud (@whonews), mantienen a sus seguidores al tanto de las últimas recomendaciones, brotes de enfermedades y nuevas estrategias de prevención. Lo mismo ocurre con el Centro para el Control de Enfermedades de Estados Unidos (@CDCgov), que informa sobre la vigilancia de brotes y el estado actual de diversas enfermedades. Además, cuenta con diversas cuentas de Twitter asociadas para temas específicos.

En el 2009, debido al brote de H1N1, se registraron más de 2 millones de tweets conteniendo los términos: swine flu, (influenza porcina), $\mathrm{H} 1 \mathrm{~N} 1$, y otras palabras relacionadas ${ }^{(5)}$. Los tweets se utilizaron para diseminar información de fuentes confiables en cuanto a prevención, así como para compartir diversas experiencias y reportar nuevos brotes ${ }^{(5)}$, lo que sugiere el uso potencial de este sitio web como herramienta de vigilancia sindrómica.

En el Perú, los esfuerzos aun son escasos, y organismos como el Instituto Nacional de Enfermedades Neoplásicas (@Neoplasicasperu), el Instituto Nacional de Salud del Niño (@insn_online), y el Ministerio de Salud (@Minsa_Peru), son algunos ejemplos del uso de Twitter en nuestro medio, para difundir información relacionada con la salud. Sin embargo, según nuestra información, ninguna institución pública o académica posee políticas de comportamiento en este medio social.

En conclusión, Twitter es actualmente un medio de información útil, de rápido crecimiento, fácil de utilizar y de gran cobertura, que ofrece múltiples oportunidades para mantener actualizados a los usuarios en temas de salud, educación e investigación. Su uso debería ser promovido activamente entre los alumnos, docentes e investigadores, especialmente aquellos relacionados con temas de ciencia y salud. Además, consideramos importante que las instituciones públicas y académicas desarrollen e implementen políticas en cuanto al uso del Twitter y otras redes sociales.

\section{AGRADECIMIENTOS}

Este trabajo fue realizado parcialmente gracias al apoyo del Proyecto QUIPU de la UPCH, un programa auspiciado por el Fogarty International Center/National Institutes of Health (FIC/NIH), proyecto: D43TW008438 y parcialmente por el proyecto R01TW007896 financiado por el FIC/NIH.

\section{Conflictos de Interés}

Los autores declaran no tener conflictos de interés en la publicación de esta carta.

\section{REFERENCIAS BIBLIOGRÁFICAS}

1. Instituto de Opinión Pública de la Pontificia Universidad Católica del Perú. Estado de la Opinión Pública: El uso de Internet a nivel Nacional. [Documento en Internet]. Lima; 2010. [Fecha de acceso: 11 febrero 2011]. Disponible en: http://www.scribd.com/doc/35873486/Uso-de-InternetJunio-2010-Nacional

2. Stieger S, Burger C. Let's go formative: continuous student ratings with Web 2.0 application Twitter. Cyberpsychol Behav Soc Netw. 2010;13(2):163-7.

3. Ebnera M, Lienhardtb C, Rohsc M, Meyerd I. Microblogs in Higher Education - A chance to facilitate informal and process-oriented learning? Comp \& Edu. 2010;55(1):92100.

4. Curioso WH, Garcia PJ, Castillo GM, Blas MM, PerezBrumer A, Zimic M, Red QUIPU. Reforzando las Capacidades en Investigación en Informática para la Salud Global en la Región Andina a través de la Colaboración Internacional. Rev Peru Med Exp Salud Publica. 2010;27(3):449-57.

5. Chew C, Eysenbach G. Pandemics in the age of Twitter: content analysis of Tweets during the 2009 H1N1 outbreak. PLoS One. 2010;5(11):e14118.
Correspondencia: Dr. Walter H. Curioso.

Dirección: Av. Honorio Delgado 430 San Martín de Porres, Lima 31, Perú.

Teléfono: (511)319-0028.

Correo electrónico: walter.curioso@upch.pe 